\title{
Évaluation des pratiques culturales de l'arachide favorisant la contamination par les aflatoxines dans trois régions de Côte d'Ivoire
}

\author{
Ama Léthicia MANIZAN ${ }^{1 *}$, David AKAKI ${ }^{2}$, Isabelle PIRO-METAYER ${ }^{3}$, \\ Didier MONTET ${ }^{3}$, Catherine BRABET ${ }^{3}$ et Rose KOFFI-NEVRY ${ }^{1}$
}

${ }^{1}$ UNA, UFR des Sciences et Technologie des Aliments, Laboratoire de Biotechnologie et Microbiologie des Aliments, 02 BP 801 Abidjan, Côte d'Ivoire rosenevry2002@gmail.com;

${ }^{2}$ INP-HB, Département Génie Chimique et Agro-alimentaire, Laboratoire des Procédés Industriels de Synthèse, de l'Environnement et des Énergies Nouvelles, BP 1313 Yamoussoukro, Côte d'Ivoire davidakaki@yahoo.fr

${ }^{3}$ UMR 95 QualiSud / Cirad/ Université of Montpellier, TA B-95 /16, 73 rue J-F Breton, 34398 Montpellier cedex 5, France.isabelle.piro-metayer@cirad.frdidier.montet@cirad.frcatherine.brabet@cirad.fr;

*Auteur correspondant ; E-mail: manizanlethicia@yahoo.fr

\section{REMERCIEMENTS}

Ce travail a été financé par le projet Européen 3CIvoire (EuropeAid/129596/L/ACT/CI DCINSAPVD/2010/64), le Service de Coopération et d'Action Culturelle de l'ambassade de France en Côte d'Ivoire et le Ministère de l'enseignement supérieur et de la recherche scientifique de Côte d'Ivoire.

\section{RESUME}

L'arachide fait partie des plantes les plus sujettes à la contamination par les aflatoxines depuis le champ, la récolte et même durant le stockage. Cette étude a été conduite avec pour objectif de déterminer les points, facteurs ou sources de contamination par les aflatoxines au cours des pratiques culturales de l'arachide en Côte d'Ivoire. Sur la base d'un questionnaire, des informations sur les producteurs et leurs pratiques culturales ont été collectées dans les régions Centre, Nord et Ouest du pays. Réalisée entièrement par les femmes dont 95,3\% n'avait aucun niveau d'étude, la culture de l'arachide diffère d'une région à une autre. Elle se cultive seule $(30,2 \%)$, en association avec d'autres cultures (maïs sorgho, mil, pistache) $(44,2 \%)$ ou en rotation $(25,6 \%)$. Les dates de semis et de récolte vont de mars à juillet dans le Centre et l'Ouest et de mai à novembre dans le Nord. L'arachide est exposée à diverses attaques par les insectes, les rongeurs, les mauvaises herbes et des maladies qui occasionnent de nombreuses pertes. Les critères de maturité sont l'aspect des feuilles dans le Centre et Nord du pays ou la taille des gousses et l'aspect des feuilles dans le Centre. Le respect des dates de semis, l'utilisation de semis sain, la pratique de la rotation ou l'association des cultures, influence le développement des moisissures et la production des aflatoxines. L'identification des pratiques culturales a permis de montrer leur implication dans la contamination de l'arachide par les aflatoxines.

(C) 2018 International Formulae Group. All rights reserved.

Mots clés: Aracfhide, aflatoxines, Côte d'Ivoire, pratiques culturales. 


\title{
Determination of peanut cultural practices increasing aflatoxins contamination in three regions of Côte d'Ivoire
}

\begin{abstract}
Peanuts are among plants most susceptible to aflatoxin contamination both in the field, after harvest and during storage. This study was conducted with the objective of determining the points or factors or sources of contamination by aflatoxins during peanut growing practices in Côte d'Ivoire. On basis of a questionnaire, information on farmers and their farming practices was collected in the central, northern and western regions of the country. Practice entirely $(100 \%)$ by women, $95.3 \%$ of whom had no level of education, peanut cultivation differs from one region to another. It is grown alone (30.2\%) in combination with other crops (maize sorghum, millet, pistachio) $(44.2 \%)$ or in rotation (25.6\%). Sowing and harvest dates are from March to July in the center and west and from May to November in the north. Peanut is exposed to various attacks by insects, rodents, weeds and diseases that cause many losses. Maturity criteria are leaf appearance in the central and northern parts of the country or pod size and leaf appearance in the center. Compliance with sowing dates, use of healthy seedlings, rotations or combination of crops influences development of molds and aflatoxins production. The identification of cultural practices has shown their involvement in contamination of peanuts by aflatoxins.
\end{abstract}

(C) 2018 International Formulae Group. All rights reserved.

Keywords: Peanuts, aflatoxins, cultural practices, Côte d'Ivoire.

\section{INTRODUCTION}

L'arachide (Arachis hypogaea L.), est cultivée dans plus de 100 pays dans les régions tropicales, sub-tropicales et tempérées chaudes (Sharma et al., 2006; Guchi et al., 2014). Elle est la $13^{\text {ème }}$ culture vivrière et $4^{\text {ème }}$ culture oléagineuse la plus importante au niveau mondial (Reddy et al., 2011). Dans une grande partie de l'Afrique subsaharienne, l'arachide est une culture importante tant pour la consommation domestique que pour le commerce (Christie et al., 2015). En effet, l'arachide représente l'une des principales cultures d'exportation au Mali, au Niger, au Nigéria et au Sénégal (Ndjeunga et al., 2006), et la légumine la plus importante dans les savanes de la Guinée, du Soudan et dans celles du Ghana (Dzomeku et al., 2009).

La production mondiale d'arachide (non décortiquée) est estimée à 45 millions de tonnes, avec plus de $90 \%$ de cette production provenant d'Asie (67\%) et d'Afrique (26\%). La Chine, l'Inde, le Nigéria et les Etats-Unis sont les quatre premiers pays producteurs mondiaux (FAOSTAT, 2013). Produite principalement dans les régions Nord et Centre de la Côte d'Ivoire (ANADER, 2009), la production d'arachide a été estimée à 88000 tonnes (FAOSTAT, 2013). L'intégralité de cette production est cependant consommée sur place et principalement sous forme de pâte d'arachide (Boli et al., 2013; Diakité et al., 2017).

La culture de l'arachide est confrontée à de nombreuses maladies causées par diverses moisissures phytopathogènes. C'est le cas de la fonte des semis (Aspergillus niger, Rhizopus stolonifer), la cercosporiose (Cercospora arachidicola) et la pourriture sèche des racines et des gousses (Rhizoctonia solani), qui réduisent le rendement et la qualité des graines, augmentant le coût de production (Yussif et al., 2014). Outre les maladies, les graines d'arachide sont fréquemment contaminées par des moisissures principalement du genre Aspergillus, responsable de la production de mycotoxines (Ouattara-Sourabie et al., 2011; Guchi, 2015).

Les mycotoxines, contaminants naturels de la chaîne alimentaire, retiennent de plus en plus, l'attention dans le monde entier, en raison des pertes économiques importantes, des 
risques pour la santé publique, due à leur présence dans les aliments (Wu, 2006; Morgavi et Riley, 2007). Les principales mycotoxines en causes dans la culture de l'arachide sont les aflatoxines. En effet, l'exposition aux aflatoxines peut conduire à l'aflatoxicose aiguë ou chronique, selon la durée et le niveau d'exposition. L'aflatoxine $\mathrm{B} 1$, la plus répandue des aflatoxines est le plus puissant cancérogène connu du foie (USAID, 2012). Les aflatoxines affectent principalement les aliments dans les régions tropicales et subtropicales du monde, où les systèmes agricoles (pratiques culturales, conditions de stockage) ne sont pas adéquates pour gérer les risques de sécurité alimentaire (Wu et Khlangwiset, 2010; Dieme et al., 2016).

Selon Cardwell et al. (2001) et aussi USAID (2012), la contamination par les aflatoxines des cultures agricoles telles que l'arachide et les céréales provoque des pertes annuelles de plus de 750 millions de Dollars en Afrique. Les gousses et les graines peuvent être contaminées par les aflatoxines dans le sol avant la récolte, pendant et après la récolte et au cours de la conservation. De nombreuses études ont montré la contamination par les aflatoxines de l'arachide au Benin (Hell et al., 2000), au Botswana (Mphande et al., 2004), au Nigeria (Ezekiel et al., 2012) au Cameroun (Abia et al., 2013) et en Côte d'Ivoire (Sangare-Tigori et al., 2006; Boli et al., 2014; Kouadio et al., 2014).

Les aflatoxines constituent un problème grave de santé qui affecte toute la chaîne alimentaire, depuis le champ jusqu'à la consommation. Actuellement, les chercheurs examinent les effets des acides naturels, des sels, et des extraits de plantes pour détoxiquer les cultures contaminées (USAID, 2012). Cependant, quelques interventions moins coûteuses, comme la rotation de culture (Ansari et al., 2015), de meilleur technique de récolte et de stockage (Degraeve et al., 2016), peuvent être misent en ouvre avant et après la récolte afin de réduire la contamination par les Aspergillus et donc par les aflatoxines. En Côte d'Ivoire très peu d'études font cas du rapport entre les pratiques culturales et la contamination par les aflatoxines. Cette étude a pour objectif de déterminer les points ou facteurs ou sources de contamination par les aflatoxines au cours des pratiques culturales de l'arachide en Côte d'Ivoire, afin de contribuer à réduire la contamination par les aflatoxines.

\section{MATERIEL ET METHODES}

Une enquête a été réalisée auprès des producteurs d'arachide dans les régions Centre (Gbêkê), Nord (Poro) et Ouest (Haut Sassandra) de la Côte d'Ivoire afin de collecter des informations sur les pratiques de la culture de l'arachide. Les villages et les producteurs ont été sélectionnés avec l'aide de coopératives agricoles des régions suscitées. L'enquête a consisté en un entretien direct avec les producteurs d'arachide sur les pratiques culturales (traitements phytosanitaires, maladies des plants), les variétés cultivées (grosse ou petite graine), les traitements post récolte (séchage, stockage), le contrôle et la gestion de la qualité du produit final et la consommation. Au total, ce sont 43 producteurs qui ont été interrogés dont 10 de la région de Bouaké (village de N'guessankro et Allèkro), 28 de Korhogo (Gbandokaha et Pokaha), 5 de Daloa (Sapia).

\section{Analyse statistique}

Les données collectées ont fait d'abord l'objet de codage puis dépouillées à l'aide du logiciel IBM SPSS Statistics Version 21. Le test de Khi-deux de Pearson, les valeurs de Phi de Pearson ont permis de déterminer la corrélation entre les variables (type de culture, traitements appliqués) analysées et les zones investiguées. Il existait une corrélation lorsque le degré de signification du Khi-deux est < 0,05. La force de cette corrélation a été déterminée par la valeur du Phi. La corrélation entre les variables analysées a été forte, lorsque Phi $>0,7$. 


\section{RESULTATS}

\section{Caractéristiques des producteurs}

Le Tableau 1 montre que l'ensemble des personnes interrogées étaient des femmes dont l'âge était supérieur ou égale à 15 ans. La tranche d'âge de la majorité $(65,1 \%)$ était située entre 30 et 60 ans. Parmi ces productrices, $95,3 \%$ n'avait aucun niveau d'étude et seulement $4,7 \%$ avait le niveau d'étude primaire. Ces productrices sont toutes $(100 \%)$ indépendantes. C'est-à-dire qu'elles travaillent de façon isolée et ont pour la plupart, en moyenne 20 ans d'expérience dans le domaine. Parmi ces dernières, seulement $11,6 \%$ ont reçu une formation sur les bonnes pratiques de production. Les producteurs d'arachides étaient des femmes, illettrées qui n'ont pour la plupart aucunes notions des bonnes pratiques de production.

\section{Pratiques culturales}

Le Tableau 2 présente les pratiques culturales en fonction des localités. À l'exception du mode de culture (semis ou germoir) et des traitements appliqués (désherbage, phytosanitaire), il existait une forte corrélation entre le type de culture, les périodes de semis et de récolte et les zones soumises à l'étude. Quelle que soit la zone investiguée, la culture de l'arachide se faisait par semis, soit en association ou en rotation avec d'autres cultures comme le maïs, le mil et le sorgho, soit sans association ni rotation. Deux grandes variétés d'arachide étaient cultivées. Ce sont notamment, la variété dite tardive produisant de grosses graines et la variété dite précoce, produisant de petites graines. La plupart des productrices s'auto fournissaient la semence ou les obtenaient sur les marchés. La variété tardive semée à partir du mois de mai est récoltée à la fin du mois d'octobre ou en novembre, principalement dans la région de Korhogo. Quant à la variété précoce, le semis se faisait dans le courant du mois de mars et la récolte dans les mois de juin et juillet, ceci, dans les régions de Bouaké et Daloa.

\section{Agents biotiques nuisibles liés à la culture de l'arachide}

Il apparait dans le Tableau 3 que les problèmes étaient relatifs à la présence de termites et de mauvaises herbes (27,9\%), suivis des insectes et l'apparition de taches noires sur les feuilles $(20,9 \%)$. La présence de rongeurs, de perdrix et l'effet de la sécheresse sont également à relever. Cependant, l'apparition de maladies, d'insectes ou d'autres nuisibles dépendait des zones investiguées. Les facteurs les plus importants relevés dans la localité de Korhogo étaient les termites et les mauvaises herbes $(42,9 \%)$ suivis des insectes, alors que les difficultés rencontrées à Daloa étaient uniquement liées à la présence des perdrix et de rongeurs (100\%). Les facteurs nuisibles dans la région de Bouaké concernaient principalement les rats palmistes, l'atrophie foliaire (70\%) et le trio rongeur, perdrix et sècheresse (30\%).

\section{Critères de maturité des plants pour la récolte}

La technique de récolte était manuelle pour l'ensemble $(100 \%)$ des productrices. Il existait une forte corrélation entre les critères de maturité et la zone investiguée. La maturité des plants et le moment de la récolte se déterminaient à partir d'un certain nombre de critères. A Korhogo, les critères étaient basés sur le séchage des feuilles. A Bouaké, ils étaient caractérisés essentiellement (100\%) par le jaunissement des feuilles, tandis qu'à Daloa, la maturité des gousses se signalait aussi bien par la taille des gousses que par l'aspect des feuilles (Tableau 4). 
Tableau 1 : Caractéristiques des productrices d'arachide dans les zones d'investigation.

\begin{tabular}{|c|c|c|}
\hline Caractéristiques & $\begin{array}{c}\text { Nombre } \\
\text { de producteurs }(n=43)\end{array}$ & Pourcentage (\%) \\
\hline \multicolumn{3}{|l|}{ Sexe } \\
\hline Masculin & 0 & 0 \\
\hline Féminin & 43 & 100 \\
\hline \multicolumn{3}{|l|}{ Age } \\
\hline $15-30$ ans & 12 & 27,9 \\
\hline $30-60$ ans & 28 & 65,1 \\
\hline$>60$ ans & 3 & 7 \\
\hline \multicolumn{3}{|c|}{ Niveau d'instruction } \\
\hline Primaire & 2 & 4,7 \\
\hline Secondaire & 0 & 0 \\
\hline Supérieur & 0 & 0 \\
\hline Aucun & 41 & 95,3 \\
\hline \multicolumn{3}{|c|}{ Type de productrice } \\
\hline Indépendant & 43 & 100 \\
\hline \multicolumn{3}{|c|}{ Nombre d'années d'expérience } \\
\hline $1-10$ ans & 15 & 34,9 \\
\hline $10-20$ ans & 15 & 34,9 \\
\hline$>20$ ans & 13 & 30,2 \\
\hline \multicolumn{3}{|l|}{ Formation reçue } \\
\hline Oui & 5 & 11,6 \\
\hline Non & 38 & 88,4 \\
\hline
\end{tabular}

Tableau 2 : Répartitions des pratiques culturales de l'arachide selon les localités.

\begin{tabular}{|c|c|c|c|c|}
\hline \multirow[t]{2}{*}{ Pratiques culturales } & \multicolumn{4}{|c|}{ Pourcentage (\%) } \\
\hline & Korhogo & Bouaké & Daloa & \% Total \\
\hline & $(n=28)$ & $(\mathrm{n}=\mathbf{1 0})$ & $(\mathbf{n}=\mathbf{5})$ & \\
\hline \multicolumn{5}{|l|}{ Type culturale (a) } \\
\hline Association & $53,6(15)$ & $40(4)$ & 0 & 44,2 \\
\hline Rotation & 0 & $60(6)$ & $100(5)$ & 25,6 \\
\hline Seule & $46,4(13)$ & 0 & 0 & 30,2 \\
\hline \multicolumn{5}{|l|}{ Mode de culture (b) } \\
\hline Semis & $100(28)$ & $100(10)$ & $100(5)$ & 100 \\
\hline Germoirs & 0 & 0 & 0 & 0 \\
\hline \multicolumn{5}{|l|}{ Variété et cycle de culture (a) Tardive } \\
\hline (grosses graines) - 6mois Précoce & $100(28)$ & 0 & 0 & 65,1 \\
\hline (petites graines) - 3 à 4 mois & 0 & $100(10)$ & $100(5)$ & 34,9 \\
\hline \multicolumn{5}{|l|}{ Mode d'obtention des semences (a) } \\
\hline Semis personnel & 0 & $70(7)$ & $100(5)$ & 27,9 \\
\hline Semis commercialisés & $53,6(15)$ & 0 & 0 & 34,9 \\
\hline Semis propres et/ou achetés & $46,4(13)$ & $30(3)$ & 0 & 37,2 \\
\hline \multicolumn{5}{|l|}{ Traitements appliqués (b) } \\
\hline Désherbage manuel (daba) & $53,6(15)$ & $100(10)$ & $100(5)$ & 69,8 \\
\hline
\end{tabular}


Désherbage manuel-herbicide

$\begin{array}{llll}46,4(13) & 0 & 0 & 30,2 \\ 100(28) & 0 & 0 & 65,1 \\ 0 & 100(10) & 100(5) & 34,9 \\ & & & \\ 0 & 20(2) & 0 & 4,7 \\ 0 & 0 & 100(5) & 11,6 \\ 0 & 80(8) & 0 & 18,6 \\ 100(28) & 0 & 0 & 65,1\end{array}$

30,2

Période de semis (a)

Mai

Mars

Période de récolte (a)

Juin

Juillet

Juin-juillet

100 (28)

$(\mathrm{n})=$ nombre d'individus $;(\mathrm{a})=$ forte corrélation entre la variable et la localité (degré de signification du Khi2 <0,05 et Phi $>70 \%)$; (b) = pas de corrélation entre la variable et la localité (degré de signification du khi2 $>0,05$ ).

Tableau 3 : Agents biotiques nuisibles à la culture d'arachide en fonction des localités.

\begin{tabular}{lllll}
\hline Agents biotiques nuisibles & \multicolumn{5}{c}{ Pourcentage (\%) } \\
\cline { 2 - 5 } & $\begin{array}{l}\text { Korhogo } \\
(\mathbf{n = 2 8 )}\end{array}$ & $\begin{array}{l}\text { Bouaké } \\
(\mathbf{n = 1 0})\end{array}$ & $\begin{array}{l}\text { Daloa } \\
(\mathbf{n = 5})\end{array}$ & \% Total \\
\hline Insectes & $21,4(6)$ & 0 & 0 & 14 \\
Insectes-tâches noires sur feuilles & $32,1(9)$ & 0 & 0 & 20,9 \\
Mauvaises herbes & $3,6(1)$ & 0 & 0 & 2,3 \\
Termites-mauvaises herbes & $42,9(12)$ & 0 & 0 & 27,9 \\
Rat palmistes- feuilles qui s'atrophient & 0 & $70(7)$ & 0 & 16,3 \\
Rongeur-perdrix-sécheresse & 0 & $30(3)$ & 0 & 7 \\
Perdrix-rongeur & 0 & 0 & $100(5)$ & 11,6 \\
\end{tabular}

(n) = nombre d'individus

Tableau 4 : Critères de maturité selon les localités.

\begin{tabular}{lllll}
\hline Critères de maturité (a) & \multicolumn{4}{c}{ Pourcentage (\%) } \\
\cline { 2 - 5 } & $\begin{array}{l}\text { Korhogo } \\
(\mathbf{n = 2 8 )}\end{array}$ & $\begin{array}{l}\text { Bouaké } \\
(\mathbf{n = 1 0})\end{array}$ & $\begin{array}{l}\text { Daloa } \\
(\mathbf{n = 5})\end{array}$ & \% Total \\
& & & & \\
\hline Feuilles sèches & $100(28)$ & 0 & 0 & 65,1 \\
Jaunissement des feuilles & 0 & $100(10)$ & 0 & 23,3 \\
Feuilles tachetées, sèches, taille des gousses & 0 & 0 & $60(3)$ & 7 \\
Feuilles tachetées, taille des gousses & 0 & 0 & $40(2)$ & 4,7
\end{tabular}

(n) = nombre d'individus ; (a) = forte corrélation entre la variable et la localité (degré de signification

du Khi $2<0,05$ et Phi $>70 \%)$; (b) = pas de corrélation entre la variable et la localité (degré de signification du khi2 >0,05). 


\section{DISCUSSION}

Les enquêtes menées dans les principales zones de production de l'arachide ont permis de déterminer les pratiques prérécolte de l'arachide en Côte d'Ivoire. La majorité des productrices $(65,1 \%)$, ont un âge compris entre 30 et 60 ans avec au moins 20 ans d'expérience et 95,3\% n'ont aucun niveau d'étude. Ce résultat pourrait s'expliquer par le fait qu'en Côte d'Ivoire, un grand nombre de villages ne sont pas dotés d'infrastructures scolaires. En plus, dans la plupart des cultures de Côte d'Ivoire, les femmes sont appelées à pratiquer les travaux domestiques et sont de ce fait, non scolarisées pour la plupart. Non reconnues comme partenaires à part entière dans la famille ou dans la société africaine, les femmes ont été privées de l'égalité d'accès à l'éducation, à la formation professionnelle, à l'emploi, aux soins de santé, à la propriété foncière et au pouvoir politique (Anunobi, 2002). Les productrices ne sont ni regroupées en coopérative, ni encadrées par des structures d'appui agricole. En effet, en Côte d'Ivoire, les structures d'Appui agricole et les coopératives agricoles sont plus axées sur l'encadrement des paysans pour les cultures de rente à fort rendement économique comme le cacao, le coton et l'Hévéa.

La culture de l'arachide varie selon la zone d'étude. Elle se fait en rotation ou en association avec d'autres cultures comme le maïs, le mil, le sorgho et le poids de terre. Ces résultats sont en cohérence avec ceux d'une étude conduite au Ghana, où $46 \%$ des producteurs font la culture de l'arachide en association avec d'autres cultures (Konlan et al., 2013; Yussif et al., 2014). Toutefois, il convient de noter qu'une parcelle peut être destinée uniquement à la culture de l'arachide. L'arachide étant très sensible à la culture précédente, il est recommandé de ne pas semer l'arachide plusieurs années consécutives sur la même parcelle. La rotation permet de faire varier la flore du sol, réduisant la masse de certaines souches pathogènes. Selon la FAO (2002) et Ansari et al. (2015), la rotation des cultures d'arachide avec d'autres cultures comme le sorgho, le maïs, le coton et le ricin permettrait une utilisation plus efficace de la fertilité résiduelle des sols.

Il ressort de ce travail, que les femmes utilisent leurs propres semences (obtenue de la récolte précédente), ou s'en procurent sur les marchés. Dans la plupart des pays africain, excepté le Sénégal, l'approvisionnement en semences est insuffisante ou très limitée (Ndjeunga et al., 2006; Hamasselbe et al., 2012). Selon Ntare et al. (2008), la semence des variétés améliorées est un intrant coûteux, en particulier pour ce qui concerne l'arachide; ce qui expliquerait que les femmes préfèrent utiliser des semences prélevées de la récolte précédente. Aucune des productrices interrogées au cours de ce travail, n'utilisait d'insecticide ou de fongicide pour traiter les semences, ce qui pourrait favoriser la contamination du sol par les moisissures. En effet, selon Ntare et al. (2008), avant le semis, les graines doivent être décortiquées et triées à la main, puis traitées avec un mélange d'insecticide et de fongicide afin de contrôler le développement des bactéries et des moisissures et de certains ravageurs présents dans le sol.

La date de semis varie selon le profil pluviométrique de la zone de culture et la durée du cycle végétatif de la variété utilisée. Dans les régions de Bouaké et Daloa, les semis s'effectuent au cours du mois de mars qui correspond au début de la saison pluvieuse dans ces zones. Selon Boko-Koidia et al. (2016), le mois de mai marque le début de la saison des pluies dans la région de Korhogo, définissant ainsi le début des semis. Comparativement, selon une étude menée au Ghana, les dates de semis se situent d'avril à juillet (Dzomeku et al., 2009). Cela pourrait s'expliquer par le fait que la Côte d'Ivoire et le Ghana se situent dans la même zone agro écologique. Le respect des périodes de semis permet de réduire la contamination par les aflatoxines. En effet, selon (Arias et al., 2014), la production des aflatoxines par Aspergillus flavus dans les arachides se fait principalement dans les graines immatures pendant la sécheresse.

Le rendement de la culture d'arachide dépend de la zone. En effet, l'arachide est 
exposée à des attaques diverses: maladies (taches foliaires, atrophie des feuilles) et ravageurs (rongeurs, perdrix) qui peuvent occasionner des pertes importantes et une détérioration de la qualité marchande de la production. De même en Afrique du sud, les maladies les plus répandues restent les taches foliaires précoces et la pourriture de la tige (South Africa Ministry, 2010). En effet, les insectes créent des ouvertures sur les graines, ce qui faciliterait le développement des moisissures au cours de la conservation. Selon Dieme et al. (2016), l'infection par les Aspergillus et la production d'aflatoxines dépendent du stress subi par la plante et des dommages causés par les insectes et les ravageurs.

La période de récolte est fonction de la zone d'étude. Elle varie des mois de juin au mois de novembre, selon la date de semis et la variété de l'arachide cultivée. Les conditions climatiques de récolte peuvent avoir un effet sur la croissance des moisissures. En effet, selon Bhat et Reddy (2017) la forte variation des températures au cours des périodes de récolte favorise le développement des moisissures et des mycotoxines dans l'arachide.

La récolte faite manuellement, se fait généralement suivant plusieurs critères de maturité. A savoir, l'aspect des feuilles (sèches, jaunissement ou apparition de tâches) et la taille des gousses. Cependant, il est difficile de déterminer la maturité des gousses en utilisant uniquement des caractéristiques foliaires, compte tenu du fait qu'elles sont produites dans le sol. En plus, les feuilles sèches et la présence de tâches foliaires peuvent être des symptômes de certaines maladies telles que la cercosporiose et la nécrose. Selon Rowland et al. (2006), la récolte prématurée entraîne des pertes dans la production. Aussi, pour Gulluoglu et al. (2016), le test le plus pertinent pour contrôler la maturité de l'arachide consiste-il en la vérification du parenchyme interne de la gousse qui, de duveteux et turgescent, devient lisse et sec, et la couleur passe de blanc (le plus immature) au jaune, orange, brun et noir (le plus mature). La contamination par les aflatoxines peut se produire si les arachides ne sont pas récoltées au temps opportun ou récoltées mécaniquement (Torres et al., 2014).

\section{Conclusion}

Les pratiques culturales dans l'ensemble diffèrent d'une région à l'autre. Le manque de connaissances des bonnes pratiques de production induit la pratique de mauvaises techniques culturales. Ce sont entre autres l'utilisation de semis non traités et l'absence de traitement fongique pouvant favoriser le développement des moisissures et donc des aflatoxines. Cependant, le respect des dates de semis, la pratique de techniques agricoles, telles que la rotation ou l'association des cultures et l'utilisation de fongicide son des moyens pouvant aider à la réduction de la contamination des graines par les aflatoxines. Ce travail a permis de mettre en évidence que les pratiques culturales de l'arachide en Côte d'Ivoire jouent un rôle important dans la contamination de l'arachide par la flore fongique et partant de là par les aflatoxines.

\section{CONFLITS D'INTERETS}

Les auteurs déclarent qu'il n'existe aucun conflit d'intérêts.

\section{CONTRIBUTIONS DES AUTEURS}

$\mathrm{CB}$ et DA ont dirigé les travaux. La collecte et l'analyse des données ont été réalisées par ALM et IP-M. La rédaction du présent document est le fait de ALM, DM et RK-N.

\section{REMERCIEMENTS}

Nos remerciements vont à l'endroit de Monsieur Kouamé De Modeste, des coopératives agricoles des régions de Korhogo, Bouaké et Daloa et de tous les producteurs qui ont donné de leur temps pour la réalisation de ces travaux.

\section{REFERENCES}

Abia WA, Warth B, Sulyok M, Krska R, Tchana AN, Njobeh PB, Dutton MF, Moundipa PF. 2013. Determination of multi-mycotoxin occurrence in cereals, nuts and their products in Cameroon by liquid chromatography tandem mass spectrometry (LC-MS/MS). Food Control $31 \quad$ (2), 438-453. DOI:10.1016/j.foodcont.2012.10.006. 
ANADER (Agence Nationale de DEveloppement Rural). 2009. La production vivrière: un enjeu national. Bulletin de liaison de l'agence nationale de développement rural. Bull. Liaison L'agence Natl. Dév. Rural 12p.

Anunobi F. 2002. Women and development in Africa: From marginalization to gender inequality. African Social Science Review, 2(1): 41-63. $\mathrm{http} / / /$ digitalcommons.kennesaw.edu/assr /vol2/iss $2 / 3$

Ansari MA, Prakash N, Punitha P, Baishya LK. 2015. Post-harvest management and value addition of groundnut. Join Dir. ICAR Res. Complex NEH Reg. Manipur Cent. Lamphelpat Imphal- 795004.

Arias RS, Sobolev VS, Orner VA, Dang PM, Lamb MC. 2014. Potential involvement of Aspergillus flavus laccases in peanut invasion at low water potential. Plant Pathol., $\quad$ 63: $\quad 354-364$. DOI:10.1111/ppa.12088.

Bhat R, Reddy KRN. 2017. Challenges and issues concerning mycotoxins contamination in oil seeds and their edible oils: Updates from last decade. Food Chem., 215 : 425-437. DOI:10.1016/j.foodchem.2016.07.161.

Boko-Koidia ANN, Cissé G, Koné B, Séri D. 2016. Variabilité climatique et changements dans l'environnement à Korhogo en Côte d'Ivoire: Mythes Ou Réalité ? Eur. Sci. J., 12(5). DOI:10.19044/esj.2016.v12n5p158.

Boli ZA, Zoue LT, Alloue-Boraud WM, Kakou CA, Koffi-Nevry R. 2013. Proximate composition and mycological characterization of peanut butter sold in retail markets of Abidjan (Côte d'Ivoire). J. Appl. Biosci., 72 : 58225829.

Boli ZA, Zoue LT, Koffi-Nevry R, Koussemon M. 2014. Fungal contamination and mycotoxins' occurrence in peanut butters marketed in abidjan district (Côte d'Ivoire). Food and Environment Safety,
8(3): 267-275.

Cardwell KF, Desjardins A, Henry SH, Munkvold G, Robens J. 2001. Mycotoxins: The Cost of Achieving Food Security and Food Quality. APS. http://www.apsnet.org/publications/apsne tfeatures/Pages/Mycotoxins.aspx

Christie ME, Kyamureku P, Kaaya A, Devenport A. 2015. Farmers, peanuts, and aflatoxins in Uganda: A gendered approach. Dev. Pract., 25 : 4-18. DOI:10.1080/09614524.2015.983459.

Degraeve S, Madege RR, Audenaert K, Kamala A, Ortiz J, Kimanya M, Tiisekwa B, De Meulenaer B, Haesaert G. 2016. Impact of local pre-harvest management practices in maize on the occurrence of Fusarium species and associated mycotoxins in two agro-ecosystems in Tanzania. Food Control, 59: 225-233. DOI:10.1016/j.foodcont.2015.05.028.

Diakité A., Gouli Bi Irie M., N'dri DK, Yapo J. 2017. Détermination de la contamination par l'aflatoxine B1 de la pâte d'arachide consommée par la population en Côte d'Ivoire: intérêt de la chromatographie sur couche mince. Int. J. Biol. Chem. Sci., 11(4): 1646-1654. DOI: http://dx.doi.org/10.4314/ijcbs.v11i4.19.

Dieme E, Fall R, Sarr I, Sarr F, Traore D, Seydi M. 2016. Contamination des céréales par l'aflatoxine en Afrique: revue des méthodes de lutte existantes. Int. J. Biol. Chem Sci., 10 (5) : 2285-2299. DOI: http://dx.doi.org/10.4314/ijcbs.v10i5.27.

Dzomeku IK, Abudulai M, Brandenburg RL, Jordan DL. 2009. Survey of Weeds and Management Practices in Peanut (Arachis hypogaea L.) in the Savanna Ecology of Ghana. Peanut Sci., 36: 165-173.

Ezekiel CN, Sulyok M, Warth B, Odebode AC, Krska R. 2012. Natural occurrence of mycotoxins in peanut cake from Nigeria. Food Control, 27: 338-342. DOI:10.1016/j.foodcont.2012.04.010. 
FAO (Food and Agriculture Organisation). 2002. UEMOA - COTE D'IVOIRE: Appui à la mise en oeuvre de la Politique agricole de l'Union en matière de sécurité alimentaire. Programme régional de sécurité alimentaire. $31 \mathrm{p}$.

FAO. 2013. FAOSTAT. Disponible sur: <http://faostat.fao.org/faostat9> (consulter le 5.10.2016)

Guchi E. 2015. Effect of Storage Time on Occurrence of Aspergillus species in Groundnut (Arachis hypogaea L.) in Eastern Ethiopia. J. Appl. Environ. Microbiol., 3(1): 1-5. DOI: 10.12691/jaem-3-1-1.

Guchi E, Ayalew A, Dejene M, Ketema M, Asalf B, Fininsa C. 2014. Occurrence of Aspergillus Species in Groundnut (Arachis hypogaea L.) along the Value Chain in Different Agro-Ecological Zones of Eastern Ethiopia. J. Appl. Environ. Microbiol., 2: 309-317. DOI: 10.12691/jaem-2-6-7

Gulluoglu L, Bakal H, Onat B, Kurt C, Arioglu H. 2016. The effect of harvesting on some agronomic and quality characteristics of peanut grown in mediterranean region (Turkey). Turk. J. Field Crops, 21: 224-232. DOI: 10.17557/tjfc.20186.

Hamasselbe A, Ntoupka M, Sale A, Njiemoun. 2012. L'analyse des semences d'arachide fermières pour le contrôle de la qualité. Int. J. Biol. chem. Sci., 6(4): 1687-1694.

DOI:

http://dx.doi.org/10.4314/ijcbs.v6i4.25.

Hell K, Cardwell KF, Setamou M, Poehling H-

M. 2000. The influence of storage practices on aflatoxin contamination in maize in four agroecological zones of Benin, west Africa. J. Stored Prod. Res., 36: 365-382.

Konlan S, Sarkodie-Addo J, Asare E, Adu-

Dapaah H, Kombiok MJ. 2013. Groundnut (Arachis hypogaea L.) varietal response to spacing in the humid forest zone of Ghana. J. Agric. Biol. Sci., 8: 642-651.

Kouadio JH, Lattanzio VMT, Ouattara D, Kouakou B, Visconti A. 2014. Assessment of mycotoxin exposure in Côte D'Ivoire (Ivory Coast) through multi-biomarker analysis and possible correlation with food consumption patterns. Toxicol Int, 21: 248-257. DOI:10.4103/0971-6580.155336.

Morgavi DP, Riley RT. 2007. An historical overview of field disease outbreaks known or suspected to be caused by consumption of feeds contaminated with Fusarium toxins. Anim. Feed Sci. Technol. 137: 201-212. DOI:10.1016/j.anifeedsci.2007.06.002.

Mphande FA, Siam BA, Taylor JE. 2004. Fungi, aflatoxins, and cyclopiazonic acid associated with peanut retailing in Botswana. J. Food Prot., 67: 96-102.

Ndjeunga J, Ntare BR, Waliyar F, Ramouch M. 2006. Groundnut seed systems in West Africa: Current practices, constraints and opportunities. http://oar.icrisat.org/5286/.

Ntare BR, Diallo AT, Ndjeunga J, Waliyar F. 2008. Groundnut seed production manual International Crops Research Institute for the Semi-Arid Tropics. http://oar.icrisat.org/id/eprint/420.

Ouattara-Sourabie PB, Nikiema PA, Traore AS. 2011. Caractérisation de souches d'Aspergillus spp isolées des graines d'arachides cultivées au Burkina Faso, Afrique de l'Ouest. Int. J. Biol. Chem. $\begin{array}{lll}\text { Sci. } & \mathbf{5}(3) . & 1232-1249 .\end{array}$ DOI:

http://www.ajol.info/index.php/ijbcs/articl e/view/72269

Reddy EC, Sudhakar C, Reddy NP.2011. Aflatoxin contamination in groundnut induced by Aspergillus flavus type fungi: A critical review. http://imsear.li.mahidol.ac.th/handle/123 456789/161664. 
Rowland DL, Sorensen RB, Butts CL, Faircloth WH. 2006. Determination of Maturity and Degree Day Indices and their Success in Predicting Peanut Maturity. Peanut Sci. 33, 125-136.

South Africa Ministry. 2010. Departement Agriculture, Forestry and Fisheries Republic of South Africa. Groundnuts, Production guidelines (24p.).

Sangare-Tigori, Moukha S, Kouadio HJ, Betbeder A-M, Dano DS, Creppy E. 2006. Co-occurrence of aflatoxin $B_{1}$, fumonisin $\mathrm{B}_{1}$, ochratoxin $\mathrm{A}$ and zearalenone in cereals and peanuts from Côte d'Ivoire. Food Addit. Contam., 23: 1000-1007. DOI: 10.1080/02652030500415686.

Sharma V, Purohit GR, Arya RS, Harsh M. 2006. Evaluation of some complete rations in sheep incorporating unconventional feed resources of arid zone in India. Anim. Nutr. Feed Technol., 6: 135-141.
Torres AM, Barros GG, Palacios SA, Chulze SN, Battilani P. 2014. Review on pre- and post-harvest management of peanuts to minimize aflatoxin contamination. Food Res. Int., 62: 11-19. DOI:10.1016/j.foodres.2014.02.023.

USAID (United States Agency for international Development). 2012. Aflatoxine: une synthèse de la recherche en santé, agriculture et commerce. Rapport final. 82p.

Wu F. 2006. A New Concept to Engineer Specific Transcription of Transplastomic Genes. ISB News Rep., 1-3.

Wu F, Khlangwiset P. 2010. Health economic impacts and cost-effectiveness of aflatoxin-reduction strategies in Africa: case studies in biocontrol and postharvest interventions. Food Addit. Contam. Part A, 27: 496-509. DOI: 10.1080/19440040903437865.

Yussif IJ, Charles K, Mahama O, Kwabena A, Yirzagla J. 2014. Farmers' Perception and Farming Practices on the Effect of Early and Late Leaf Spots on Groundnut Production in Northern Ghana. J. Biol. Agric. Healthc., 4 : 22-28. 